\title{
A Cross-Lagged Panel Study of Dissociation and Posttraumatic Stress in a Treatment-Seeking Sample of Survivoors of Childhood Sexual Abuse
}

\author{
Siobhan Murphy, ${ }^{1}$ Ask Elklit, ${ }^{1}$ Jamie Murphy, ${ }^{2}$ Philip Hyland ${ }^{3}$ and Mark Shevlin ${ }^{2}$ \\ ${ }^{1}$ University of Southern Denmark \\ ${ }^{2}$ Ulster University \\ ${ }^{3}$ National College of Ireland
}

Objective: The current prospective study assessed the temporal relations between dissociation and posttraumatic stress (PTS) in a sample of treatment-seeking female survivors of childhood sexual abuse. PTS refers to symptoms associated with posttraumatic stress disorder (PTSD) in the absence of a clinical diagnosis of PTSD. Method: Initial assessment was on average 23 years after the onset of abuse $(N=405)$, and participants were followed-up after 6 months $(N=245)$ and 12 months $(N=119)$. Results: Findings indicated that dissociative experiences and PTS were highly correlated within each wave of data collection. Cross-lagged panel analysis revealed that at each assessment period dissociative symptoms and PTS levels, respectively, were primarily explained by scores on the same variable at the previous assessment period. Although further reciprocal relations between dissociation and PTS were evident, these associations were relatively weak in magnitude. Conclusion: Current results provide important insights into the temporal relations between dissociative symptoms and PTS. The high correlations between dissociative experiences and PTS several years after trauma exposure have important clinical implications that may affect their treatment and trauma recovery. (C) 2017 Wiley Periodicals, Inc. J. Clin. Psychol. 73:1370-1381, 2017.

Keywords: childhood sexual abuse (CSA); posttraumatic stress; dissociative symptoms; cross-lagged analysis; temporal ordering

Extant literature has demonstrated the associations between dissociation and posttraumatic stress disorder (PTSD), but there is significant variability across study design, type of dissociation measured, and sample composition (Carlson, Dalenberg, \& McDade-Montez, 2012; van der Hart, van Ochten, van Son, Steele, \& Lensvelt-Mulders, 2008). Dissociation has been predominantly measured in two ways; peritraumatic dissociation (often referred to as state dissociation) refers to dissociative symptoms that occur during or in the immediate aftermath of a traumatic event; persistent dissociation (or trait dissociation) refers to the tendency to dissociate across the lifespan (Fleming \& Resick, 2016). The distinction between these different types of dissociation and their relationship with PTSD is an important consideration in traumatology research because studies examining the association between both types of dissociation are inconsistent. For example, peritraumatic dissociation has been reported as a strong risk factor for PTSD (Breh \& Siedler, 2007), although evidence from prospective studies do not support these findings (Briere, Scott, \& Weathers, 2005).

Prospective studies that have included measures of both peritraumatic and persistent dissociation have found persistent dissociation was a stronger predictor of PTSD over time (Briere et al., 2005; Murray, Ehlers, \& Mayou, 2002; Werner \& Griffin, 2012). These studies raise important implications for the understanding of the dissociation-PTSD relationship because the risk of PTSD appears less about whether dissociation occurred during or in the

Please address correspondence to: Siobhan Murphy, National Centre of Psychotraumatology, Department of Psychology, University of Southern Denmark. E-mail: smurphy@health.sdu.dk 
acute aftermath of the traumatic experience than the persistence of dissociative experiences over time.

Evidence from treatment studies have found baseline dissociation levels were significantly associated posttreatment dissociation, PTSD, and depression (Lynch, Forman, Mendelsohn, \& Herman, 2008). Notably, when predicting PTSD or depression, the amount of variance explained by baseline dissociation as compared to initial PTSD or depression was relatively small. These findings suggest baseline dissociation has attenuated effects on posttreatment levels of PTSD in comparison to PTSD and depression. Brand and Stadnik (2013) reported change in dissociation predicted changes in PTSD in a treatment-seeking sample of individuals with a dissociative disorder diagnosis. However, PTSD was not a significant predictor of change in dissociation; rather, only baseline dissociation significantly predicted change in dissociation. The authors argue that a possible explanation for this finding was that the therapists in this particular study may have focused more intensely on dissociation given their specialized training in treatment of dissociative disorders.

Further, using data from a randomized control trial of cognitive processing therapy (CPT) Resick, Suvak, Mitchell and Iverson (2012) examined if dissociation predicted PTSD treatment outcome in a sample of females exposed to sexual and/or physical abuse. Results indicated that dissociation affected the treatment conditions differently, in that women who endorsed low pretreatment dissociation responded most efficiently to cognitive therapy, whereas those with high levels of dissociation, specifically, depersonalization, responded better to CPT, which included written accounts of the trauma. This finding highlights that the combination of written accounts and cognitive therapy was more effective for individuals with high levels of dissociative symptoms. The authors propose that written accounts in CPT may support reconstruction of the fragmented memory and help place the event in context, which in turn assists in emotional processing of the trauma (Resick et al., 2012). Consequently, assessing for dissociative symptoms in treatment planning has important implications in deciding the optimal type of therapy for the individual.

Less research has examined whether PTSD influences later dissociative symptoms. In one study, Zerach, Greene, Ginzburg, and Solomon (2014) examined persistent dissociation and PTSD in a Veteran sample across three time points spanning from 18 to 35 years postwar. They found PTSD mediated the relationship between war captivity and persistent dissociation across the three time points. Notably, when looking at the independent effects of the PTSD symptom clusters, intrusion was the strongest predictor of dissociation. A possible explanation for this finding is that intrusive symptoms are associated with a greater lack of control, which could be argued to evoke stronger dissociative reactions (Zerach et al., 2014).

Alternatively, other evidence suggests dissociative features represent a more severe form of PTSD in a minority of individuals. Support for this proposition is reflected by the recent inclusion of a dissociative-PTSD subtype in the Diagnostic and Statistical Manual of Mental Disorders (DSM-5; American Psychiatric Association, 2013). Numerous studies have supported the presence of a dissociative-PTSD subtype with prevalence estimates ranging from $6 \%$ to 25.4\% (Armour, Karstoft, \& Richardson, 2014; Stein et al., 2013; Steuwe, Lanius, \& Frewin, 2012; Wolf et al., 2012). These studies have found that individuals with dissociative-PTSD are more commonly exposed to complex forms of trauma, such as child sexual abuse (CSA) or war exposure. Many clinical and neurobiological factors have also been identified that differentiate this subtype from "pure" PTSD and non-PTSD populations (Lanius et al., 2010; Wolf et al., 2012).

Finally, an important consideration relates to the sample under investigation. Empirical findings indicate higher rates of dissociation and PTSD have been reported in individuals experiencing certain types of trauma, particularly, survivors of CSA (Chu, Frey, Ganzel, \& Matthews, 1999; Kilcommons \& Morrison, 2005; Koopman et al., 2003; Zlotnick et al., 1994). This has led research to examine a number of sexual trauma factors that may contribute to higher rates of dissociative and PTSD symptoms. Studies examining abuse-specific characteristics have consistently found that the number of perpetrators, abuse duration, and incest are associated with higher dissociative symptoms (Elliot \& Briere, 1992; Gold, Hill, Swingle, \& Elfant, 1999). Conversely, other factors such as age at abuse onset have produced discrepant results. Zlotnick 
et al. (1994) reported significant associations between age of abuse onset and higher dissociative symptomatology; however, when the number of perpetrators were included in the analysis, this finding was no longer significant.

Other studies report age of abuse onset predicts different outcomes, for example, CSA onset before age 12 predicted diagnostic criteria for major depression, while those abused after the age of 12 were 10 times more likely to develop PTSD (Schoedl et al., 2010). This indicates that the developmental stage abuse occurs may be an important indicator of posttraumatic outcomes. However, not all studies support the effect of age of abuse onset, as demonstrated in a metaanalysis of 37 studies that found no effect of age on the relationship between CSA and adult psychopathology (Paolucci, Genuis, \& Violato, 2001). Given these inconsistencies, it is therefore important to examine the unique and shared effects of abuse-specific characteristics on both dissociation and PTSD in CSA survivors.

Collectively, the clinical, empirical, and conceptual links between dissociation and PTSD have been extensively examined. A limitation in the literature is that few studies have focused on the temporal and dynamic interplay between posttraumatic stress (PTS) and dissociative symptoms years or even decades after the trauma exposure. Consequently, how these constructs interact and influence each other over time remains equivocal. The current study intends to build upon the existing literature by using cross-lag panel models whereby dissociative symptoms and PTS are considered together, which makes it possible to formally test for reciprocal predictive relationships.

To our knowledge, only one study (Marshall \& Snell, 2002) has assessed reciprocal temporal relations between PTSD symptoms and peritraumatic dissociative experiences using this analytical framework. They found high correlations between dissociative experiences and PTSD symptoms across three waves of data collection (days after assault, 3- and-12-month assessment intervals) in a sample of physical assault survivors. However, baseline reports of peritraumatic dissociative experiences were not predictive of follow-up PTSD symptom severity after controlling for baseline PTSD. Therefore, this study will further contribute to the literature by examining the dissociation-PTSD relationship decades after trauma exposure. Importantly, recognizing how these constructs interact over time is critical in clinical practice and treatment because both dissociative and PTSD symptoms require targeted treatment interventions that, when unrecognized, may hamper the treatment response and recovery (Lanius, Brand, Uermetten, Frewen, \& Spiegal, 2012; Price, Kearns, Houry, \& Rithbaum, 2014; Spitzer, Barnow, Freyberger, \& Grabe, 2007).

The aim of the current study is to prospectively assess the temporal interplay between dissociative symptoms and PTS decades after traumatic exposure using a treatment-seeking sample of adult survivors of CSA. The study was predicated on the following hypotheses. First, dissociation and PTS will be highly correlated within assessment periods. Second, consistent with previous findings (e.g., Briere et al., 2005; Murray et al., 2002), dissociation may predict the longitudinal course of PTS. Alternatively, consistent with findings by Zerach and colleagues (2014), PTS may influence the longitudinal course of dissociation. It is also possible there is a bidirectional effect whereby PTS and dissociation will reciprocally influence each other over the three assessment periods. The second aim was to include age- and abuse-specific characteristics (i.e., age of abuse onset, duration of abuse, number of abusive acts) to determine if these risk factors have shared or unique effects on PTS and dissociative symptoms.

\section{Method}

\section{Participants and Procedure}

The participants were consecutive female outpatients $(\mathrm{N}=405)$ at four (now three regional) treatment centers in Denmark. The treatment centers operate for individuals who were sexually abused in childhood and are supported by the Ministry of Social Affairs. Ethical approval for use of data gathered from this sample was obtained from the relevant university ethical boards in Denmark. Exclusion criteria in the treatment centers were (a) evidence of intoxication at time of visit, (b) a diagnosis of a psychotic disorder, (c) self-harming behavior, (d) engagement in 
treatment elsewhere, and (e) diagnosis of a personality disorder. Excluded clients are referred to either specialized institutions or affiliated volunteer centers for treatment. All attendees presented with distress and impairment resulting from their traumatic abuse history and received individual psychotherapy of an eclectic nature that suited their needs. When clients initially attended the treatment center, they were asked to fill out a number of questionnaires during their first session; therapy was planned based on these questionnaires. The therapist shared the findings with the client during the following session. The present study is based on information from the questionnaires.

The mean age of the sample was 35.85 years (standard deviation $[S D]=10.83$; range 15 to 70 years) and all participants were Caucasian. Of the participants, $51 \%$ were married or cohabiting. The average age the abuse started at was $6.60(S D=4.89)$ and lasted on average 7.6 years $(S D=6.37)$. Only $8.8 \%$ of the participants reported they had experienced a single abuse act, with the mean number of different abusive acts being $3.36(S D=1.34)$. The mean number of years since their sexual abuse ended was $23.71(S D=12.34)$. Participants were assessed for PTSD (T1: $\mathrm{N}=405)$ after they had received the first treatment session. The participants were reassessed again 6 months later $(\mathrm{T} 2: \mathrm{N}=245)$, and finally 12 months later $(\mathrm{T} 3: \mathrm{N}=119)$.

\section{Measures}

Trauma Symptom Checklist (TSC; Briere \& Runtz, 1989). This self-report measure comprises 35 items rated in relation to the previous month ("How often have you experienced each of the following in the last month"). The response format is rated on a 4-point Likert-type scale $(1=$ no, $2=$ yes sometimes, $3=$ yes often, $4=$ yes very often $)$. The TSC measures seven constructs: Depression, Anxiety, Dissociation, Sleep Disturbances, Somatisation, Interpersonal Sensitivity, and Aggression. The revised version of the TSC 35 has undergone psychometric testing (Elklit \& Brink, 2003). The current study used the dissociation subscale, which includes seven items measuring, for example, memory problems, feeling that things are "unreal," and "feeling as though you are not in your body." Total possible scores range from 7 to 28 , with higher scores indicative of higher levels dissociative symptoms. The dissociation subscale demonstrated acceptable reliability in the current sample, with Cronbach's alpha $(\alpha=.72-.83)$ across the three waves of data collection.

The Harvard Trauma Questionnaire Part IV (HTQ; Mollica, et al., 1992). This self-report measure includes 16 items designed to assess the DSM-IV PTSD symptoms. The response format is rated on a 4-point Likert-type scale ranging from 1 (not at all) to 4 (all the time). To establish a more conservative measure of PTS, a symptom was rated as present if the item corresponding to the symptoms scored 3 or 4 , recoded to 1 , and item responses scoring 1 or 2 were recoded to 0 ; summed scores were used for the main analysis; and total possible scores ranged from 0 to 16, with higher scores indicative of higher levels of PTS severity. The Danish version of the HTQ has been used in a wide range of trauma populations, with reports of good reliability and validity (Bach, 2003). Mollica et al. (1992) reported 88\% concordance between those endorsing symptoms consistent with PTSD diagnostic criteria based on the HTQ and a diagnostic interview assessing PTSD. The reliability of the total scale was satisfactory $(\alpha=.66-.87)$ across the three waves of data collection.

\section{Analysis}

This study employed cross-lagged panel analysis (e.g., Kessler \& Greenberg, 1981) to determine the temporal relations between the dissociation and PTS across three time points. Robust maximum likelihood estimation (Yuan \& Bentler, 1998) was used to allow parameters to be estimated using all available information. This method is considered superior to alternative methods of dealing with missing data, such as listwise deletion (Schafer \& Graham, 2002). All cross-lagged panel analyses were conducted in Mplus 6.1 (Muthen \& Muthen, 2010).

The aim of cross-lagged panel analysis is to find the most parsimonious model solution. The analysis proceeds in three linked phases. First, a model that includes lagged-effects and no 
cross-lagged paths is tested. Within-wave residual correlations are also included. The second step compares the fit of the model with no cross-lagged effects to a model in which cross-lagged effects are introduced. If the model with no cross-lagged effects is supported, then this provides evidence that there are no temporal cross-lagged effects. If the model with cross-lagged paths is supported, then the analysis progresses to a third step. The cross-lagged paths are constrained equal and this model is compared to the model in which the cross-lagged effects are not constrained equal. If the model with freely estimated cross-lagged effects provides superior fit, then this is evidence that there is a dominant variable in the model driving temporal relations (Schell, Marshall, \& Jaycox, 2004). Alternatively, if the model with constrained cross-lagged effects is supported, then it suggests there is no single dominant variable in the model that determines temporal relations. Once the most appropriate model is determined, regression coefficients are inspected.

Model fit was determined using standard procedures: a non-significant chi-square $(\chi 2)$ test; comparative fit index and Tucker Lewis index values greater than .90 reflect acceptable model fit, and values greater than .95 reflect excellent model fit; root-mean-square error of approximation with $90 \%$ confidence intervals and standardized root-mean-square residual values of .05 or less reflect excellent model fit, while values less than .10 reflect acceptable model fit. Furthermore, the Akaike information criterion, the Bayesian information criterion, and the sample size-adjusted Bayesian information criterion are used to evaluate alternative models, with the smaller value in each case indicating the best fitting model.

\section{Results}

\section{Attrition Analysis}

The attrition rate in the current study was poor, with approximately only $30 \%$ of the baseline sample participating in each assessment period. Regretfully, we did not have information on participants' reasons for leaving the service; however, because therapy was based on an individual approach, it may be speculated that participants felt they no longer needed the service. Nevertheless, because of these high attrition rates, we conducted a series of independent samples $t$ tests to examine differences between those who completed each assessment period and those who left the service. No significant differences were found across the three assessment periods in terms of age, abuse onset, abuse duration, number of abuse acts, dissociation, and PTS.

\section{Descriptive Statistics}

The correlations, means, and standard deviations for dissociation and PTS levels are presented in Table 1. Across the three time points there were small declines in levels of dissociative experiences. Levels of PTS severity reduced moderately over the three time points. Correlations among the three measures of PTS were high, ranging from .63 to .67. Correlations among the measures of dissociative symptoms were similarly high, ranging from .52 to .66. Correlations across PTS and dissociative symptoms given in the same assessment (i.e., within wave) were also high, ranging from .62 to .80 .

Given the wide variation in years since sexual abuse ended, additional analyses were conducted to examine the effects of this variable. A categorical variable representing time since abuse ended was computed, which grouped individuals based on the categories: (a) younger than 10 years, (b) younger than 20 years, and (c) older than 20 years. A series of one-way analyses of variance were then conducted. Results indicated that individuals who experienced abuse within the past 10 years had significantly higher levels of baseline dissociation (mean $[M]=17.27, S D=3.78$, eta squared $=0.07)$ and PTS $(M=11.86, S D=2.79$, eta squared $=0.03)$ than individuals in both the younger than 20 years dissociation $(M=15.35, S D=4.24)$ and PTS $(M=10.57$, $S D=3.26)$ and older than 20 years dissociation $(M=14.16, S D=4.05)$ and PTS $(M=10.37$, $S D=3.43$ ) groups. Despite reaching significance, the actual differences in scores were small to moderate, as evidenced by the effect sizes, with no further significant differences in either variable after the baseline assessment. 
Table 1

Correlations and Descriptive Statistics for PTS and Dissociative Symptoms at Baseline, 6-Month, and 12-Month Assessments

\begin{tabular}{lcccccc}
\hline & Diss_T1 & PTS_T1 & Diss_T2 & PTS_T2 & Diss_T3 & PTS_T3 \\
\hline Diss_T1 & - & & & & & \\
PTSD_T1 & $.62^{* *}$ & - & & & & \\
Diss_T2 & $.52^{* *}$ & $.43^{* *}$ & - & & & \\
PTSD_T2 & $.35^{* *}$ & $.51^{* *}$ & $.70^{* *}$ & - & & \\
Diss_T3 & $.66^{* *}$ & $.56^{* *}$ & $.76^{* *}$ & $.63^{* *}$ & - & \\
PTSD_T3 & $.53^{* *}$ & $.63^{* *}$ & $.60^{* *}$ & $.67^{* *}$ & $.80^{* *}$ & - \\
Means & 14.94 & 10.76 & 12.81 & 8.18 & 11.84 & 6.62 \\
SD & 4.28 & 3.27 & 4.29 & 4.58 & 4.10 & 4.95 \\
N & 377 & 405 & 232 & 245 & 113 & 119 \\
\hline
\end{tabular}

Note. PTS $=$ posttraumatic stress; Diss_T1 = Dissociation Time $1 ;$ PTSD_T1 = PTSD Time 1; Diss_T2 = Dissociation Time 2; PTSD_T2 = PTSD Time 2; Diss_T3 = Dissociation Time 3; PTSD_T3 = PTSD Time 3.

${ }^{* *} \mathrm{p}<.001$.

Table 2

Model Fit Statistics of Cross Lagged Panel Analysis

\begin{tabular}{|c|c|c|c|c|c|c|c|c|c|}
\hline Model & $x^{2}$ & $\mathrm{df}$ & CFI & TLI & RMSEA & SRMR & AIC & BIC & ssaBIC \\
\hline 1 & $48.76^{*}$ & 24 & .96 & .93 & $\begin{array}{l}.05 \\
(.03, .07)\end{array}$ & .10 & 15551.87 & 15716.23 & 15586.13 \\
\hline 2 & $32.31^{*}$ & 20 & .98 & .96 & $\begin{array}{l}.04 \\
(.01, .06)\end{array}$ & .06 & 15541.11 & 15721.50 & 15578.71 \\
\hline 3 & $32.83^{\mathrm{ns}}$ & 23 & .98 & .97 & $\begin{array}{l}.03 \\
(.00, .06)\end{array}$ & .06 & 15536.72 & 15705.09 & 1557.82 \\
\hline
\end{tabular}

Note. $\chi 2=$ chi-square; $\mathrm{df}=$ degrees of freedom; $\mathrm{CFI}=$ comparative fit index; $\mathrm{TLI}=$ Tucker Lewis index; RMSEA = root mean square error of approximation; SRMR = standardized root mean square residual; $\mathrm{AIC}=$ Akaike information criterion; $\mathrm{BIC}=$ Bayesian information criterion; ssaBIC $=$ sample size-adjusted $\mathrm{BIC}$.

${ }^{*} \mathrm{p}=<.05$.

\section{Cross-Lagged Panel Analysis}

Table 2 presents the results from these analyses. Age, abuse onset, abuse duration, and number of abuse acts were included as covariates. In the first step of the analysis (Model 1), a model with no cross-lagged paths (autoregressive effects) was tested. This model was then compared to a fully cross-lagged model (Model 2), which prospectively shows if dissociative symptoms predict PTS and PTS predicts dissociative symptoms (reciprocal effects), which resulted in a moderately improved model fit. In Model 3, cross-lagged paths were subsequently constrained equal, and this model was superior to the model in which the cross-lagged effects were freely estimated, suggesting there was not one dominant variable influencing future symptom levels. Regression weights from this model were therefore assessed.

Figure 1 presents auto-regression weights, represented by single-headed arrows. The results show that age was significantly associated with PTSD. The results further indicate that for dissociative experiences, the auto-regressive effects were all moderate to strong, ranging from .47 to $.61(\mathrm{p}<.001)$. Similar auto-regression weights were found for PTS across the three time points .41 to $.60(\mathrm{p}<.001)$. The cross-lagged effects, represented by diagonal single-headed arrows in Figure 1, however, were weak, ranging from .11 to $.16(\mathrm{p}<.005)$. These results suggest that there are temporal relations between dissociative symptoms and PTS, although of a weak magnitude. The primary factor, however, explaining the dissociative symptoms and PTS levels 


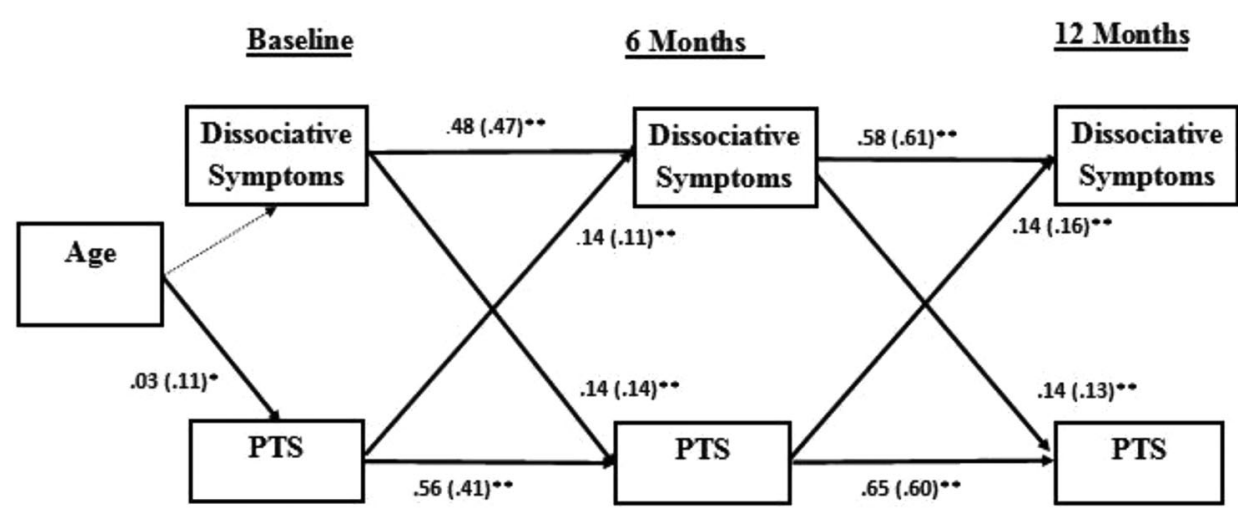

Figure 1. Unstandardised path coefficients for the final cross-lagged panel model (standardized path coefficients in parentheses).

Note. Dashed lines indicate nonsignificant paths.

$*=\mathrm{p}<.05, * * \mathrm{p}<.001$.

at the 6 and 12-month assessment periods, respectively, are scores on the same variable from the previous time point (e.g., dissociation levels at 6 months is the best predictor of dissociation levels at 12 months; PTS at 6 months is the best predictor of PTS levels at 12 months). The results also revealed that among the covariate variables, only age was a significant positive predictor of PTS.

\section{Discussion}

The primary aim of the present study was to examine the temporal relations between dissociative symptoms and PTS in a female, treatment-seeking sample exposed to CSA. The results overall indicated there were decreases in both dissociative symptoms and PTS severity across the three time points. This finding was not unexpected because the participants in the current sample are undergoing therapy for the childhood sexual abuse and supports previous treatment-seeking studies (Lynch et al., 2008). Further, these findings are consistent with Brand and Stadnik's (2013) study of a treatment-seeking sample of individuals with a dissociative disorder diagnosis; they found that changes in initial dissociation were associated with changes in PTS. These findings indicate that improvement in one symptomatic area is associated with improvements in another.

In line with our first hypothesis, PTS and dissociative symptoms were strongly correlated at each assessment, notably, these correlations strengthened over the 12-month assessment period. This indicates that a very strong relationship emerged between PTS and dissociative symptoms decades after trauma exposure. This finding is consistent with studies indicating the strong positive relationship between PTSD and dissociation and the dissociative nature of some PTSD symptoms (Brand \& Stadnik, 2013; Carlson et al., 2012). A possible explanation for this finding is that some females who were continuing to report high levels of PTS and dissociative symptoms 6 to 12 months after treatment may represent individuals with the dissociative-PTSD subtype (Steuwe et al., 2012; Wolf et al., 2012).

Further, these studies have found that this subgroup appears to be characterized by females who have experienced sexual trauma. This may have important treatment implications because evidence suggests that individuals who experience high levels of dissociation and PTSD respond better to phase-orientated treatment approaches (Steele, Van Der Hart, \& Nijenhuis, 2005). This type of treatment involves an initial focus on reducing symptom severity through skills training in emotion regulation, followed by working through traumatic memory using techniques such as narrative storytelling (Cloitre, Petkova, Wang, \& Lassell, 2012). This explanation is speculative, however, and would require further investigation before firm conclusions can be made.

The current findings lend weak support for our second and third hypotheses because at each assessment period dissociative symptoms and PTS levels, respectively, were primarily explained 
by scores on the same variable at the previous assessment. Dissociative symptoms displayed a slightly stronger temporal effect than PTS. The results further demonstrated these effects were stronger between Time 2 and Time 3. Cross-lagged effects indicated that dissociative experiences were a significant predictor of posttraumatic symptoms over time, supporting prospective studies indicating that dissociation is related to PTS severity (Briere et al., 2005; Murray et al., 2002; Werner \& Griffin, 2012). PTS was also a significant predictor of dissociative symptoms across the three waves of data collection. Notably, the effects in the current study were weak and their initial scores at the previous assessments better explained both dissociative symptoms and PTSD symptoms. This finding is supported by the model fit indices, which demonstrated only slight improvements in model fit at each stage of the analysis, therefore suggesting that the cross-lagged effects make little improvement to the model.

It is plausible to speculate that the weakened cross-lagged effects may be the result of a stronger interaction between dissociative symptoms and PTS in the short term, posttrauma period; however, after a longer period of time, the predictive utility of these two constructs are attenuated. This is evidenced by the stronger association between baseline dissociation and PTS and subsequent levels of the same variable at each assessment period. This partially supports previous findings that reported change in dissociation over time was predicted by baseline dissociation rather than PTSD (Brand \& Stadnik, 2013). Similar findings were reported in Lynch and colleagues (2008) study in which posttreatment PTSD was largely explained by baseline PTSD rather than baseline dissociation. Subsequently, targeting dissociative and PTS symptoms separately in therapy may have greater effectiveness in reducing the symptomatology of each construct over time.

Nevertheless, despite the attenuated effects, it is noteworthy that reciprocal relations between PTS and dissociative symptoms remained. These findings are inconsistent with those reported in Marshall and Snell's (2002) study. A possible explanation relates to the type of dissociation assessed. Dissociative symptoms that manifest during trauma may arise from states such as hyperventilation, panic, or arousal (Bryant et al., 2011), and therefore such symptoms may not indicate risk of dissociative symptoms after the trauma (Dalenberg et al., 2012). Further, peritraumatic dissociation features strongly in a diagnosis for acute stress disorder (ASD: Bryant, 2007), rather than PTSD, which may be another mechanism to explain the inconsistent findings between different types of dissociation and PTSD. Importantly, Resick and colleagues (2012) noted that the majority of studies measuring dissociation are based on the Dissociative Experiences Scale (Bernstein \& Putnam, 1986), which is designed to assess characteristics of dissociative disorders. The measures used in the current study and in Resick's study represent trait-like dissociative symptoms with traumatic characteristics and therefore more closely aligned with PTSD than dissociative disorders (Resick et al., 2012), which may further explain the discrepant research findings.

Finally, in examining a range of abuse-specific characteristics, the results indicated that age of onset, duration, and number of abuse acts were not significant predictors of either dissociation or PTSD. These findings are consistent with a meta-analysis that found CSA was significantly associated with PTSD; however, abuse-specific outcomes (i.e., age, gender, perpetrator-relationship, type of abuse and frequency of abuse) were not significant (Paolucci et al., 2001). These findings also support a recent study by Bak-Klimek et al. (2014), who reported nonsignificant associations between specific abuse characteristics and psychopathological disorders in CSA survivors attending a specialist psychotherapy service. One possible explanation for these nonsignificant findings is that there may be other factors that affect survivors of CSA psychological outcomes that were not included in this study. For example, the co-occurrence of other types of childhood abuse and parental and peer support have been implicated as strong factors in subsequent psychopathology in adulthood (Adams \& Bukowski 2007; Chartier, Walker, \& Naimark, 2010).

Recent advances in neurodevelopmental studies have indicated certain abuse characteristics may affect different brain regions during vulnerable developmental periods. Andersen and colleagues (2008) found evidence, based on volumetric magnetic resonance imaging scans, that CSA at different stages of development had differential effects on certain brain regions and pathological outcomes. For example, abuse during the ages of 3 and 5 years was associated with depression, while PTS was associated only with abuse occurring at ages 9 and 10. Therefore, 
the effects of abuse on certain brain functions, neuroendocrine responses to stress, and immune system function at different developmental stages may be another mechanism that explains the inconsistencies in the literature regarding the effects of specific abuse characteristics and specific psychopathological outcomes.

\section{Limitations}

The current study should be considered in light of several limitations. First, the analysis was based on a treatment-seeking sample of Danish female survivors of CSA and therefore limits generalizability to other traumatised populations and different ethnic groups. Individuals with mental health and personality disorders and substance misuse problems were excluded from the study, which may have important implications for the generalizability of the findings within the wider context of survivors of sexual abuse. These exclusion criteria may also have attenuated the severity of the outcomes investigated in this study, and therefore replication using a less restricted sample is clearly warranted. Second, because the average time since trauma exposure was 23 years, participants were not assessed during the acute phases of the traumatic response. It would therefore be interesting to replicate this study using a similar traumatized population with a shorter follow-up period as a control group.

Third, the attrition rate was poor, but analyses indicated that this was not the result of the demographic and outcomes measured in the current study. Finally, Noll, Horowitz, Bonanno, Trickett, and Putman (2003) found that dissociation is not only a consequence of past trauma but also associated with vulnerability to revictimization because it prevents the individual from engaging in self-protective behaviors. Therefore, because this study did not control for revictimization, it is difficult to ascertain whether the current results may be attributed to other variables not included in the analysis. Future research could explore such variables as mediators and/or moderators of the cross-lagged effects.

\section{Conclusion}

Despite these limitations, the current study contributes valuable information on the temporal relations between dissociative symptoms and symptoms of PTS. The findings suggest that there is a strong prospective relationship between dissociative symptoms and PTS, as evident from the robust bivariate correlations. Further, although the predictive validity of these constructs was evident, effects were relatively weak. These findings are interesting for a number of reasons. First, this is the only known study that has examined the bidirectional relationship between PTS symptoms and dissociation many years after trauma exposure. Second, these findings have important clinical implications because individuals who continue to report high levels of PTS may also experience dissociative experiences with traumatic characteristics that might affect their treatment and trauma recovery.

Additionally, because the current sample included females seeking treatment for CSA, another important implication was the type of therapy provided. Therapy was based on an individual basis and therefore of an eclectic nature, so the reductions in PTS and dissociative symptoms occurred regardless of a specific type of therapy used to address these particular symptoms. However, if a phase-orientated approach of treatment focusing on dissociative and PTSD symptomatology had been provided during the initial treatment sessions, it is possible that higher reduction in these symptoms would have occurred. Finally, the findings underline the need for further prospective studies to understand the relationship between these two constructs across different trauma populations and thus inform more tailored treatment approaches.

\section{References}

Adams R. E., \& Bukowski W. M. (2007) Relationships with mothers and peers moderate the association between childhood sexual abuse and anxiety disorders. Child Abuse \& Neglect 31, 645-656.

American Psychiatric Association. (2013). Diagnostic and statistical manual of mental disorders. Washington, DC: Author. 
Andersen, S. L., Tomada, A., Vincow, E. S., Valente, E., Polcari, A., \& Teicher, M. H. (2008). Preliminary evidence for sensitive periods in the effect of childhood sexual abuse on regional brain development. American Journal of Neuropsychiatry \& Clinical Neurosciences, 20, 292-301.

Armour, C., Karstoft, K. I., \& Richardson, J. D. (2014). The co-occurrence of PTSD and dissociation: Differentiating severe PTSD from dissociative-PTSD. Social Psychiatry and Psychiatric Epidemiology, 49, 1297-1306.

Bach, M. (2003). En empirisk belysning og analyse af "Emotionel Numbing" som eventuel selvstændig faktor i PTSD [An empirical illumination and analysis of emotional numbing as an independent factor in PTSD]. Psykologisk Studieskriftserie, 6, 1-19.

Bak-Klimek, A., Karatzias, T., Elliott, L., Campbell, J., Pugh, R., \& Laybourn, P. (2013). Nature of child sexual abuse and psychopathology in adult survivors: Results from a clinical sample in Scotland. Journal of Psychiatric and Mental Health Nursing, 21, 550-557.

Bernstein, E. M., \& Putnam, F. W. (1986). Development, reliability, and validity of a dissociation scale. The Journal of Nervous and Mental Disease, 174, 727-735.

Brand, B. L., \& Stadnik, R. (2013). What contributes to predicting change in the treatment of dissociation: Initial levels of dissociation, PTSD, or overall distress? Journal of Trauma \& Dissociation, 14, 328341.

Breh, D. C., \& Seidler, G. H. (2007). Is peritraumatic dissociation a risk factor for PTSD? Journal of Trauma \& Dissociation, 8, 53-69.

Briere, J., \& Runtz, M. (1989). Trauma Symptom Checklist (TSC-33). Journal of Interpersonal Violence, 4, $151-163$.

Briere, J., Scott, C., \& Weathers, F. (2005). Peritraumatic and persistent dissociation in the presumed etiology of PTSD. American Journal of Psychiatry, 162, 2295-2301.

Bryant, R. A. (2007). Does dissociation further our understanding of PTSD? Journal of Anxiety Disorders, 21, 183-191.

Bryant, R. A., Brooks, R., Silove, D., Creamer, M., O’Donnell, M., \& McFarlane, A. C. (2011). Peritraumatic dissociation mediates the relationship between acute panic and chronic posttraumatic stress disorder. Behaviour Research and Therapy, 49, 346-351.

Carlson, E. B., Dalenberg, C., \& McDade-Montez, E. (2012). Dissociation in posttraumatic stress disorder part I: Definitions and review of research. Psychological trauma: Theory, Research, Practice, and Policy, 4, 479-489.

Chartier, M. J., Walker, J. R., \& Naimark, B. (2010). Separate and cumulative effects of adverse childhood experiences in predicting adult health and health care utilization. Child Abuse \& Neglect, 34, 454-464.

Chu, J. A., Frey, L. M., Ganzel, B. L., \& Matthews, J. A. (1999). Memories of childhood abuse: Dissociation, amnesia, and corroboration. American Journal of Psychiatry, 156, 749-755.

Cloitre, M., Petkova, E., \& Wang, J. (2012). An examination of the influence of a sequential treatment on the course and impact of dissociation among women with PTSD related to childhood abuse. Depression and Anxiety, 29, 709-717.

Dalenberg, C., \& Carlson, E. B. (2012). Dissociation in posttraumatic stress disorder part II: How theoretical models fit the empirical evidence and recommendations for modifying the diagnostic criteria for PTSD. Psychological Trauma: Theory, Research, Practice, and Policy, 4, 551-599.

Elklit, A., \& Brink, O. (2003). Acute stress disorder in physical assault victims visiting a Danish Emergency Ward. Violence and Victims, 18, 461-472.

Elliott, D. M., \& Briere, J. (1992). Sexual abuse trauma among professional women: Validating the Trauma Symptom Checklist-40 (TSC-40). Child Abuse \& Neglect, 16, 391-398.

Fleming, C. E., \& Resick, P. A. (2016). Predicting three types of dissociation in female survivors of intimate partner violence. Journal of Trauma \& Dissociation, 17, 267-285.

Gold, S. N., Hill, E. L., Swingle, J. M., \& Elfant, A. S. (1999). Relationship between childhood sexual abuse characteristics and dissociation among women in therapy. Journal of Family Violence, 14, 157-171.

Kessler, R. C., \& Greenberg, D. F. (1981). Linear panel analysis: Models of quantitative change. New York: Academic Press.

Kilcommons, A. M., \& Morrison, A. P. (2005). Relationships between trauma and psychosis: an exploration of cognitive and dissociative factors. Acta Psychiatrica Scandinavica, 112, 351-359.

Koopman, C., Sephton, S., Abercrombie, H. C., Classen, C., Butler, L. D., Gore-Felton, C., ... Spiegel, D. (2003). Dissociative symptoms and cortisol responses to recounting traumatic experiences among childhood sexual abuse survivors with PTSD. Journal of Trauma \& Dissociation, 4, 29-46. 
Lanius, R. A., Brand, B., Vermetten, E., Frewen, P. A., \& Spiegel, D. (2012). The dissociative subtype of posttraumatic disorder: Rationale, clinical and neurobiological evidence, and implications. Depression and Anxiety, 29, 701-708.

Lanius, R. A., Vermetten, E., Loewenstein, R. J., Brand, B., Schmahl, C., Bremner, J. D., \& Spiegel, D. (2010). Emotion modulation in PTSD: Clinical and neurobiological evidence for a dissociative subtype. American Journal of Psychiatry, 167, 640-647.

Lynch, S. M., Forman, E., Mendelsohn, M., \& Herman, J. (2008). Attending to dissociation: Assessing change in dissociation and predicting treatment outcome. Journal of Trauma \& Dissociation, 9, 301319.

Marshall, G. N., \& Schell, T. L. (2002). Reappraising the link between peritraumatic dissociation and PTSD symptom severity: Evidence from a longitudinal study of community violence survivors. Journal of Abnormal Psychology, 111, 626-636.

Mayer, L., \& Carrol, S. (1987). Testing for lagged, cotemporal and total dependence in cross-lagged panel analysis. Sociological Methods and Research, 16, 187-217.

Mollica, R. F., Caspi-Yavin, Y., Bollini, P., Truong, T., Tor, S., \& Lavelle, J. (1992). The Harvard Trauma Questionnaire: Validating a cross-cultural instrument for measuring torture, trauma and posttraumatic stress disorder in Indochinese refugees. The Journal of Nervous and Mental Disease, 180, 111-116.

Murray, J., Ehlers, A., \& Mayou, R. A. (2002). Dissociation and post-traumatic stress disorder: Two prospective studies of road traffic accident survivors. The British Journal of Psychiatry, 180, 363 368.

Muthén L., \& Muthén, B. (2010). Mplus User Guide. Version 7. Los Angeles: Statmodel.

Noll, J. G., Horowitz, L. A., Bonanno, G. A., Trickett, P. K., \& Putnam, F. W. (2003). Revictimization and self-harm in females who experienced childhood sexual abuse results from a prospective study. Journal of Interpersonal Violence, 18, 1452-1471.

Paolucci, E. O., Genuis, M. L., \& Violato, C. (2001). A meta-analysis of the published research on the effects of child sexual abuse. The Journal of Psychology, 135, 17-36.

Price, M., Kearns, M., Houry, D., \& Rothbaum, B. O. (2014). Emergency department predictors of posttraumatic stress reduction for trauma-exposed individuals with and without an early intervention. Journal of Consulting and Clinical Psychology, 82, 336-341.

Resick, P. A., Suvak, M. K., Johnides, B. D., Mitchell, K. S., \& Iverson, K. M. (2012). The impact of dissociation on PTSD treatment with cognitive processing therapy. Depression and Anxiety, 29, 718 730.

Schell, T. L., Marshall, G. N., \& Jaycox, L. H. (2004). All symptoms are not created equal: The prominent role of hyperarousal in the natural course of posttraumatic psychological distress. Journal of Abnormal Psychology, 113, 189-197.

Schafer, J. L. \& Graham, J. W. (2002) Missing data: Our view of the state of the art. Psychological Methods, 7, 147-177.

Schoedl, A. F., Costa, M. C. P., Mari, J. J., Mello, M. F., Tyrka, A. R., Carpenter, L. L., \& Price, L. H. (2010). The clinical correlates of reported childhood sexual abuse: an association between age at trauma onset and severity of depression and PTSD in adults. Journal of Child Sexual Abuse, 19, 156-170.

Spitzer, C. S., Barnow, S., Freyberger, H. J., \& Grabe, H. J. (2007). Dissociation predicts symptom-related treatment outcome in short-term inpatient psychotherapy. The Australian and New Zealand Journal of Psychiatry, 41, 682-687.

Steele, K., Van Der Hart, O., \& Nijenhuis, E. R. (2005). Phase-oriented treatment of structural dissociation in complex traumatization: Overcoming trauma-related phobias. Journal of Trauma \& Dissociation, 6, $11-53$.

Stein, D. J., Koenen, K. C., Friedman, M. J., Hill, E., McLaughlin, K. A., Petukhova, M., \& Kessler, R. C. (2013). Dissociation in posttraumatic stress disorder: Evidence from the world mental health surveys. Biological Psychiatry, 73, 302-312.

Steuwe, C., Lanius, R. A., \& Frewen, P. A. (2012). Evidence for a dissociative subtype of PTSD by latent profile and confirmatory factor analyses in a civilian sample. Depression and Anxiety, 29, 689-700.

van der Hart, O., van Ochten, J. M., van Son, M. J., Steele, K., \& Lensvelt-Mulders, G. (2008). Relations among peritraumatic dissociation and posttraumatic stress: A critical review. Journal of Trauma \& Dissociation, 9, 481-505.

Werner, K. B., \& Griffin, M. G. (2012). Peritraumatic and persistent dissociation as predictors of PTSD symptoms in a female cohort. Journal of Traumatic Stress, 25, 401-407. 
Wolf, E. J., Miller, M. W., Reardon, A. F., Ryabchenko, K. A., Castillo, D., \& Freund, R. (2012). A latent class analysis of dissociation and posttraumatic stress disorder: Evidence for a dissociative subtype. Archives of General Psychiatry, 69, 698-705.

Yuan, K. H., \& Bentler, P. M. (2000). Three likelihood-based methods for mean and covariance structure analysis with nonnormal missing data. Sociological Methodology, 30, 165-200.

Zerach, G., Greene, T., Ginzburg, K., \& Solomon, Z. (2014). The relations between posttraumatic stress disorder and persistent dissociation among ex-prisoners of war: A longitudinal study. Psychological Trauma: Theory, Research, Practice, and Policy, 6, 99-108.

Zlotnick, C., Begin, A., Shea, M. T., Pearlstein, T., Simpson, E., \& Costello, E. (1994). The relationship between characteristics of sexual abuse and dissociative experiences. Comprehensive Psychiatry, 35, 465-470. 